\section{Transcription Levels of Two Actin Genes (SmAct and SmAct2), Cytochrome C Oxidase Subunit II (SmCOXII), Triosephosphate Isomerase (TPI), and a Putative Translation Regulatory Protein EIF-5 during the First Seven Days of in vitro Development of Schistosoma mansoni Schistosomula}

\section{Guilherme Oliveira $^{+}$, Solange Busek, Rodrigo Corrêa-Oliveira}

Laboratório de Imunologia Celular e Molecular, Centro de Pesquisas René Rachou-Fiocruz, Av. Augusto de Lima 1517, 30190-002 Belo Horizonte, MG, Brasil

Key words: schistosomula - maturation - RNA - actin - cytochrome c oxidase subunit II - triosephosphate isomerase - EIF-5

After penetration into the mammalian host, the Schistosoma mansoni cercariae transforms into schistosomula. This immature larval form of the parasite migrates via the circulatory system of the host through the lungs, reaching the hepatic portal system where it matures and couples. A number of morphologic, behavioral and metabolic changes that take place during the development of the parastie have been well characterized. However, the understanding of the molecular mechanisms that regulate these changes at the gene level are still limited. In this paper we describe attempts we have undertaken to better understand some of these mechanisms.
During penetration through the skin of the mammalian host the cercaria looses its tail and the glycocalix that surrounds its body. The tegument of the newly transformed schistosomula undergoes further modifications. Observations made by transmission electron microscopy indicate that the trilaminate tegumental membrane of the schistomulum becomes gradually heptalaminate (D Hockley 1973 Int J Parasitol 3: 13-25). As observed by scanning electron microscopy, soon after penetration the ventral sucker is retracted into the body, where it remains until the parasites arrive at the portal system (J Crabtree 1980 Parasitology 81: 553564). After two days a regression of the spines in the mid-body and a change in the spination of the mouth are observed. By the third day, the central portion of the body is almost totally devoid of spines. The protruding tegumentary ridges on the apical region of the body and the raised basal bulbs are no longer visible. The tegument at this stage becomes pitted. At about day 14 after penetration the spines have redeveloped. The annular ridges of the pitted tegument have become interconnected by longitudinal septae. An enlargement of the mouth is observed and the oral sucker becomes more apparent. At day 18 the ventral sucker has increased in size and protrudes from the body surface.

After penetration, the body of the schistosomulum starts to contract and extend rhythmically (Crabtree loc. cit.). These movements proceed until the worm reaches the hepatic portal system, and have been associated with the facilitation of worm migration through the capillaries in the lung and systemic organs. The reduction in movement is accompanied by a decrease in maximum length. It has also been postulated that the changes that occur in body shape prevent the escape of the schistosomulum from the hepatic portal system, trapping the parasites in this site.

Several metabolical and physiological changes are detected after transformation of the parasite from cercariae to schistosomula. It has been demonstrated that transferrin and platelet derived growth factor stimulate in vitro growth and development of schistosomula (L Clemens $1989 \mathrm{~J}$ Parasitol 75: 417-421). Hormones have also been demonstrated to play an important role in parasite maturation. In this context, it has been shown that the production of ecdysteroids present in the maturing worm peak at day 6 after transformation $(\mathrm{G}$ Torpier 1982 Parasitology 84: 123-130). Serotonin has been shown to not affect the activity levels of adenylate cyclase in cercariae, in contrast to the developing parasite and adult worms where an increased level of the enzyme is observed in the presence of serotonin (M Kasschau 1982 Nature 296:
${ }^{+}$Corresponding author. Fax: +55-31-295.3566. Received 4 May 1998 Accepted 31 August 1998 
66-68). An important change that occurs after transformation is in the mode of energy metabolism of the schistosomula. Cercariae have been shown to function aerobically with carbon dioxide being generated in the Krebs cycle. However, after transformation a transition to a more anaerobic metabolism occurs (B van Oordt 1989 Parasitology 98: 409-415). This switch is triggered by the loss of the tail during the transformation process (W Von Kruger 1978 Comp Biochem Physiol 60B: 4-46) The activity of the enzyme lactic dehydrogenase remains constant in the maturing worm until it reaches the hepatic portal system. At this stage the activity levels increases exponentially (J Lawson 1980 Parasitology 81: 325-336). This and other evidence indicate that after maturation the worms appear to be mostly fermenters (E Bueding 1982 J Parasitol 68: 208-212). The levels of protein synthesis have also been shown to increase after transformation (R Harrop 1993 Parasitology 107: 265-274). Studies on the composition of the membranes of the cercariae and schistosomula have shown that they differ significant in their protein (J Kusel 1972 Parasitology 65: 55-69) and lipid composition (F Rumjanek 1981 Mol Biochem Parasitol 3: 239-252).

The levels of expression of some genes or proteins have been shown to differ during the life cycle of the parasite. Antigens expressed on the cercarial surface, schistosomula and eggs have been shown to contain cross-reactive carbohydrate epitopes, but individual protein antigens are different among the life-cycle stages, including the adult worm (A Simpson 1990 Parasitol Today 6: 40-45). There are a number of examples of genes that are trancriptionally regulated during the life cycle. A serine protease that is released during skin penetration was detected in the cercariae, but the RNA message for this protein was present only in the intramolluscan stage of development (Z Fishelson 1992 Exp Parasitol 75: 87-98). Messenger RNA derived from the genes coding for paramyosin, myosin, HSP70 and 14-3-3 were detected in sporocysts and adult worms, but not cercariae, in contrast to a gene coding for a calcium binding protein that was present preferentially in cercariae $(\mathrm{Z}$ Grossman 1990 Exp Parasitol 70: 62-71, D Schechtman 1995 Mol BiochemParasitol 73: 275278).

Some understanding of the mechanisms of gene regulation in S. mansoni have emerged more recently. Nuclear extracts of males and females have been shown to bind in a distinct way, by band-shift assays, to a region of the female specific transcript F-10 containing putative regulatory elements (A Giannini 1995 Parasitology 110: 155-161). Functional analysis of promoter elements of the
SmGST-28 gene detected the presence of proteins that bound to an AP-1 site and CAAT motifs (E Serra 1996 Mol Biochem Parasitol 83: 69-80).

The work described herein was carried out in order to further understand, at the level of gene expression, the mechanisms at work during the development of schistosomula. In this study our objective was to investigate the levels of transcripts for five genes, SmAct (actin), SmAct2 (actin), SmCOXII (cytochrome C oxidase subunit II), TPI (triosephosphate isomerase), and SmEIF-5 (putative elongation factor 5). Both actin genes (G Oliveira 1995 Mol Biochem Parasitol 75: 119-122) and the TPI gene (M dos-Reis 1993 Mol Biochem Parasitol 59: 235-242) have been previously described. Clones for the genes SmCOXII and SmEIF-5 were obtained by immunoscreening an expression cDNA library with pooled sera from mice infected for four weeks with S. mansoni.

In order to investigate the levels of transcripts for the above genes, RT-PCR was carried out carefully controlling the amount of starting RNA, reaction components, annealing temperature and cycle number of amplifications. These conditions allowed us to perform semi-quantitative studies by quantifying digitized images of ethidium bromide stained polyacrylamide electrophoresis gels. Each experiment was repeated at least four times. In the first set of experiments cercariae were transformed into schistosomula and at $2 \mathrm{hr}$ intervals, for a period of $24 \mathrm{hr}$, samples were collected and processed. The results show that the levels of transcripts for SmAct, SmAct2, SmCOXII and TPI display little fluctuation during this period. The levels of transcripts for SmEIF-5 showed a gradual increase during the first $4 \mathrm{hr}$ of culture, remaining constant thereafter.

In another set of experiments, samples were taken at $24 \mathrm{hr}$ intervals after transformation for a period of seven days. The levels of transcripts during these time points were compared with that of male and female adult worms. The levels of transcripts of SmCOXII and TPI showed little variability during this period. However, the levels of transcripts for TPI were much lower in adult females when compared with males or the developing schistosomula. The levels of SmEIF-5 transcripts were increased after $24 \mathrm{hr}$ of culture and remained constant subsequently. Adult males and females showed relatively the same levels of that of schsitosomula after $24 \mathrm{hr}$ of culture. The levels of transcript for SmAct remained constant throughout the culture period. However, the levels found in adult females were much lower than that of males and maturing worms. The levels of SmAct 2 transcript were much higher after the second day of culture, and decreased to the initial level at later 
time points, rising again at day 7 of culture. As for SmAct the levels found in mature females were lower than that of males or schistosomula.

The levels of transcripts for the above genes were also studied in eggs and cercarial bodies and tails. We observed that the levels of TPI were higher in adult male worms when compared to eggs. In contrast the levels of transcripts for SmAct, SmAct2, and SmEIF-5 were higher in eggs than in adult males. Transcript levels of SmCOXII were similar for all of the stages tested. Cercarial bodies showed higher levels of transcripts for TPI, SmEIF-5, and SmAct2 then cercarial tails. The levels of transcript for SmCOXII and SmAct were similar and cercarial bodies and tails.

The apparently unregulated transcription of the SmCOXII gene that codes for a protein that participates in energy obtention pathway in the mitochondria is quite interesting in view of the apparent lack of importance of aerobic metabolism to the maturing schistosomula (Van Oordt loc. cit.). However, the presence of this transcript does not indicate that it is necessarily being translated, or that the translated protein is part of a functioning metabolic pathway.

During the development of the schistosomula there is a change in the shape of the organism and spination of the body (Crabtree loc. cit.), as described above. These changes may be associated to changes on the usage of actin isoforms, and ultimately at the transcriptional activity of these genes. We observed changes on the levels of transcripts of both actin genes. Their transcript levels fluctuated during the first $24 \mathrm{hr}$ of culture. Two peaks of transcript levels for SmAct2 were ob- served, at 2 and 7 days of culture. Both of these genes also showed a higher level of transcription in adult males. Putative upstream regulatory elements have been identified in the gene for SmAct. These elements are the common CAAT and TATA elements, and E-box and CArG box which are involved in muscle specific transcription in other organisms (Oliveira loc.cit.). These regulatory sequences may be involved in the differential regulation of transcription of these genes, and experiments are underway to test this hypothesis.

It is known that as quickly as $3 \mathrm{hr}$ after transformation the rate of protein synthesis in the schistosomula is increased (Harrop 1993 loc. cit.). We have observed that transcript levels of the putative elongation factor SmEIF-5 were higher after transformation. These levels remained high until the parasite reached the adult stage. It is noteworthy that eggs were also shown to contain high levels of this transcript, possibly indicating a high rate of protein synthesis of this stage of development.

In conclusion our work showed that there is differential regulation of gene expression in the schistosomula. SmEIF-5 was regulated as early as $2 \mathrm{hr}$ after transformation. We also showed that both actin genes are differentially regulated. This difference may be due to known regulatory elements found upstream of the coding region of these genes. Levels of transcripts for SmCOXII were the most constant of all, and were found to be present in all tested stages of the parasite life cycle. Therefore, the lack of a described unregulated housekeeping gene, and the results described here indicate that transcripts for this gene could be used as a control for future gene expression studies. 
218 Schistosomula RNA Expression Levels - Guilherme Oliveira et al. 